\title{
LA ESCRITURA ACADÉMICA: PERCEPCIONES DE ESTUdiantes DE CiENCIAS Humanas Y CIENCIAS DE LA INGENIERÍA DE UNA UNIVERSIDAD CHILENA
}

\author{
academic Writing: Perceptions of Students of Human Sciences \\ and Engineering SCiences at a Chilean University \\ L'ÉCRITURE ACADÉMIQUE: PERCEPTIONS DES ÉTUDIANTS EN SCIENCES HUMAINES \\ ET SCIENCES DE L'INGÉNIERIE D'UNE UNIVERSITÉ CHILIENNE
}

\author{
Ana E. Vine-Jara \\ Doctora en Lingüística, Universidad \\ de Concepción. \\ Profesora asistente del \\ Departamento de Español, Facultad \\ de Humanidades y Arte, Universidad \\ de Concepción, Chile. \\ E-mail: avine@udec.cl \\ https://orcid. \\ org/0000-0002-2646-5303
}

\begin{abstract}
RESUMEN
En este artículo se presenta un estudio cuantitativo de tipo transeccional exploratorio, cuyo objetivo principal es conocer la percepción de dos grupos de estudiantes de una universidad chilena sobre las distintas dimensiones de la escritura académica. El primero proviene de Ciencias Humanas (20 estudiantes de Traducción) y el segundo, de Ciencias de la Ingeniería (32 estudiantes de Ingenierías). Para ello, se diseñó y aplicó un cuestionario tipo Likert, basado en una escala para la identificación de problemáticas en la escritura académica y en las cinco dimensiones que abarcan el antes, el durante y el después de la escritura académica (planificación, textualización, revisión, postergación del inicio de la escritura, actitudes hacia la escritura). Los resultados muestran que los alumnos de Ciencias de la Ingeniería perciben mayores dificultades para enfrentar la escritura que los de Ciencias Humanas. En las dimensiones de textualización y de revisión también se observan diferencias estadísticamente significativas entre ambos grupos. Esto evidencia la necesidad de enseñar a escribir en la universidad de forma diferenciada en los distintos ámbitos especializados.
\end{abstract}

Palabras clave: escritura académica; enseñanza de la escritura; escritura académica en ciencias humanas; escritura académica en ciencias de la ingeniería.

\section{Abstract}

This paper presents an exploratory cross-sectional quantitative study, the main goal of which was to know the perceptions about academic writing from two groups of student at a Chilean university. The first group was enrolled in a program in the field of human sciences (20 students in the Translation program) and the second was enrolled in Engineering Sciences (32 Engineering students). For this purpose, a Likert questionnaire was designed and applied, based on a scale to identify problems in academic writing and on the five dimensions covering the before, during and after academic writing ((planning, textualization, revision, postponing writing, 
attitudes towards writing). The results show that students of Engineering Sciences perceive greater difficulties in writing than students from Humans Sciences. In the dimensions of textualization and revision, statistically significant differences were also observed between both groups. This demonstrates the need to teach writing in different specialized fields at the university.

Keywords: academic writing; teaching writing; academic writing in human sciences; academic writing in engineering.

\section{RÉSUMÉ}

Cet article présente une étude quantitative de type transversale exploratoire visant principalement à connaître la perception sur l'écriture académique parmis deux groupes d'étudiants d'une université chilienne. Le premier groupe provenant d'une filière en sciences humaines (20 étudiants de traduction) et le second groupe en sciences de l'ingénierie (32 étudiants d'ingenierie). À ce but, un questionnaire de type Likert a été conçu et appliqué, basé sur une échelle d'identification des problèmes d'écriture académique dans les cinq dimensions qui couvrent l'avant, pendant et après l'écriture académique (planification, textualisation, révision, report du début de l'écriture, attitudes envers l'écriture). Les résultats montrent que les étudiants de sciences de l'ingénierie ont plus de difficultés en écriture académique. Les différences statistiques les plus significatives entre les deux groupes ont été observées pour la textualisation et la révision. Cela démontre la nécessité d'enseigner à écrire à l'université, dans les différents domaines spécialisés.

Mots-clés : écriture académique; enseignement de l'écriture; rédaction académique en sciences humaines; rédaction académique en sciences de l'ingénierie. 


\section{Introducción}

En el ámbito universitario existe la creencia de que los estudiantes que ingresan a la educación superior cuentan con las competencias suficientes para enfrentar la escritura de distintos tipos de textos (Errázuriz, Arriagada, Contreras y López, 2015). Sin embargo, la experiencia y la investigación evidencian lo contrario, pues los estudiantes, durante la educación básica y secundaria, se han dedicado a "reproducir el conocimiento", mientras que en la universidad se espera que los alumnos sean capaces de "transformar el conocimiento" a partir de lo que escriben (Scardamalia y Bereiter, 1992, p. 46). Es esta exigencia la que detona innumerables problemáticas (Errázuriz et al., 2015), que demuestran la necesidad de que este proceso sea enseñado (Carlino, 2005).

Ortiz (2011) identifica dos enfoques bajo los cuales se han realizado los trabajos o estudios sobre la escritura académica: el enfoque cognitivo y el enfoque sociocognitivo.

Según Castelló (2002), el enfoque cognitivo tiene en cuenta los procesos mentales que la escritura implica. Esta visión hizo que la atención investigativa se centrara en el proceso, más que en el producto, por lo que era necesario enseñar a escribir. En este contexto se sitúan los trabajos pioneros de Flower y Hayes (1981) y de Bereiter y Scardamalia (1987). El modelo de Flower y Hayes (1981) plantea que la escritura es una actividad recursiva y no lineal, por lo que el escritor puede volver sobre sus ideas, evaluarlas o agregar otras. Este modelo señala tres procesos básicos: planificación, textualización y revisión, a partir de los cuales se agregan tres subprocesos en la planificación: la generación de ideas, la fijación de objetivos, la formulación de las ideas en un lenguaje visible (escribir las ideas), y dos subprocesos en la revisión: la evaluación y la revisión de la producción escrita. Por su parte, Bereiter y Scardamalia (1987) reconocen dos modelos de escritura: el primero, de "decir el conocimiento", donde el escritor escribe lo que sabe sobre un tema sin una planificación (escritor inmaduro) y, el segundo, de "transformar el conocimiento", en el cual el escritor analiza la situación retórica de la composición (escritor maduro), es decir, toma en cuenta el propósito, a quién va dirigido el texto, etc.

Respecto al enfoque sociocognitivo, en este también se reconocen los procesos cognitivos que presenta la actividad de escribir, pero se enfatiza en que ellos dependen del contexto en el que se sitúa quien escribe. En palabras de Castelló:

[...] la concepción sociocognitiva supone considerar el proceso de composición escrita como un proceso cognitivo que ocurre dentro de la cabeza del escritor, pero situado socialmente; lo que acaba haciendo ese escritor depende de la situación comunicativa específica que le lleva a escribir. (2002, p. 151)

La investigación en el ámbito de la escritura ha puesto énfasis en los niveles de enseñanza básica y secundaria por sobre la universitaria. De hecho, se cree que un estudiante que finaliza la educación secundaria tiene las competencias suficientes y, por lo tanto, está preparado para elaborar distintos escritos de manera óptima (Errázuriz et al., 2015). Sin embargo, diversos estudios demuestran las dificultades a las que se enfrentan los estudiantes universitarios (Avilán, 2004; Errázuriz et al., 2015; Tapia, 2014) y la necesidad de que en los estudios superiores se enseñe a escribir desde las disciplinas.

En este artículo se presenta un estudio cuantitativo transeccional exploratorio, cuyo objetivo es conocer las percepciones de dos grupos de estudiantes de una universidad chilena, uno de las Ciencias Humanas (Traducción) y otro de las Ciencias de la Ingeniería (Ingeniería) sobre las distintas dimensiones (planificación, textualización, revisión, postergación del inicio de la escritura, actitudes hacia la escritura) del proceso de escritura académica.

\section{Marco teórico}

En este apartado, primero se introduce el concepto de alfabetización académica; luego se dan a conocer las principales dificultades que se presentan en la escritura académica (antes, durante y después del proceso) y, finalmente, se da a conocer la escala de evaluación de las concepciones sobre la escritura en la que se basa este estudio. 


\section{Alfabetización académica}

Según Carlino, el concepto de alfabetización académica comprende el "conjunto de nociones y estrategias necesarias para participar en la cultura discursiva de las disciplinas así como en las actividades de producción y análisis de textos requeridas para aprender en la universidad" (2003, p. 409). Se trata de enseñar a los estudiantes universitarios las prácticas discursivas de la disciplina para que puedan acceder y participar de ella. Enseñar sobre una disciplina es un proceso dificultoso, porque implica que los estudiantes aprenden a leer y a producir textos de una densidad compleja, en los que se abordan temáticas propias de la disciplina de formación del estudiante.

La alfabetización no es una habilidad básica que se logra de una vez y de forma definitiva; por el contrario, se trata de un proceso. En este sentido, alfabetizar académicamente implica que en cada una de las áreas se esté dispuesto a enseñar la cultura de la disciplina que se imparte, es decir, las convenciones respecto de cómo se escribe o lee en cada una de ellas. De esta manera, se logrará un verdadero proceso de inclusión de los estudiantes en sus respectivos ámbitos disciplinares. Por lo tanto, no se puede alfabetizar académicamente en una única materia ni en un solo ciclo educativo, pues cada disciplina tiene sus particularidades (Carlino, 2003).

\section{Las dificultades de la escritura}

La escritura es una actividad compleja, de operaciones interdependientes, fuertemente vinculadas a percepciones sobre el proceso de escritura, así como también a la creencia de autoeficacia de quien escribe (Difabio, 2013). Según Narvaja, Di Stefano y Pereira (2013), en la tarea de escribir influyen las características propias de la escritura y los factores cognitivos e histórico-sociales del escritor. En el contexto académico, la escritura es una actividad que exige un elevado nivel de procesamiento cognitivo, pues el alumno debe ser capaz de autorregular su propio aprendizaje (Bangert-Drowns, Hurley y Wilkinson, 2004). Esto significa que hace uso de estrategias cognitivas y metacognitivas durante el proceso de escritura.
Las dificultades de la escritura se deben a su carácter diferido, esto es, el escrito debe ser comprensible y autónomo, pues el escritor no tendrá en frente a sus posibles lectores para explicar lo que quiso decir en su texto. Narvaja et al. (2013) señalan que el hecho de que la comunicación escrita se presente de manera diferida lleva a que quien escribe tenga que ponerse en el lugar del lector (imaginarlo, tener en cuenta su contexto). Al respecto, Errázuriz et al. (2015) afirman que los estudiantes universitarios no llegan a este nivel de madurez que les permita ponerse en el lugar del lector. En palabras de Scardamalia y Bereiter (1992), no logran "transformar el conocimiento" en el desarrollo de la escritura, esto es, que su escritura ocurre sin llevar a cabo un proceso metacognitivo (planificación, pensar en los destinatarios del texto, monitoreo del proceso de escritura, etc.).

En relación con las dificultades en la escritura, Carlino (2004) ha identificado cuatro problemáticas que afectan el proceso de escritura de los universitarios. Se trata de: (1) la dificultad para escribir teniendo en cuenta el lector al que se dirige el texto; (2) el desaprovechamiento del potencial epistémico que tiene la escritura (la situación retórica de composición); (3) la tendencia a revisar los aspectos superficiales del texto y no a modificar las ideas, y (4) la postergación del momento del inicio de la escritura.

Por su parte, Rodríguez y García (2015), en el contexto de las disciplinas pedagógicas, sitúan las problemáticas de escritura en tres momentos: antes, durante y después de la escritura:

- Antes de la escritura. Los problemas se identifican en cuatro aspectos: (1) la falta de claridad respecto de los objetivos que guían la escritura, lo cual incluye tener en cuenta a los probables lectores; (2) el desconocimiento de las tipologías textuales características de los textos académicos; (3) la ausencia de una planificación de la escritura y (4) los aspectos emocionales relacionados con la tarea de escribir, por ejemplo, la inseguridad personal.

En relación con las tipologías o secuencias textuales, es preciso señalar que los textos nunca son 
puros (un cuento no solo es narración, sino que también incluye descripción), por lo que la noción de tipología textual permite organizar y clasificar la dimensión heterogénea de la mayoría de los textos. Comúnmente, se distinguen cinco secuencias textuales al interior de los textos: diálogo, narración, descripción, exposición y argumentación (Montolío, 2000). Hay algunos autores que reducen este número de tipologías. En el cuestionario que se aplicó a la muestra, a modo de ejemplo o recordatorio, solo se mencionan tres tipologías entre paréntesis.

- Durante la escritura. Las dificultades en esta etapa se relacionan con: (1) la complejidad para presentar distintas posturas en el texto; (2) la construcción de una voz propia (desapegarse de lo que dicen los otros autores, emitir su propia voz a partir de lo que ha leído sobre un tema) dentro del texto y el diálogo con los autores revisados; (3) la ausencia de un lenguaje científico característico de la disciplina; (4) la falta de evidencias que sustenten la información redactada, y (5) la complejidad para concluir el escrito.

- Después de la escritura. Las problemáticas están centradas en: (1) el apresuramiento para difundir el escrito, sin hacer reformulaciones; (2) la ausencia de parámetros que guíen la revisión del texto y que aporten a una nueva versión del mismo; (3) la dificultad para incluir los comentarios que realizan los revisores o lectores del texto.

Si bien se trata de resultados obtenidos en el ámbito específico de las disciplinas pedagógicas, estos se pueden extrapolar también a otros contextos disciplinares.

\section{La escala de evaluación de las concepciones sobre la escritura}

Según Difabio (2012), las concepciones o percepciones corresponden a las creencias o conocimientos que tienen los estudiantes en cuanto al proceso de escribir. Difabio $(2012 ; 2013)$ diseñó una escala para la evaluación de la escritura académica a nivel de posgrado, la cual se aplicó a doctorandos del área de educación.
Esta escala recoge ítems de las propuestas de cinco instrumentos (Lavelle y Bushrow, 2007; Lavelle y Guarino, 2003; Lonka, 1996; Torrance, Thomas y Robinson, 1994; Zimmerman y Bandura, 1994) y la autora agregó catorce ítems, de acuerdo con su objetivo investigativo: el posgrado.

En total, la escala propuesta se compone de setenta ítems, distribuidos en cuatro dimensiones: estrategias de escritura, estrategias de regulación, concepciones sobre la escritura y autoeficacia para la escritura. Las "estrategias de escritura" comprenden la secuencia de procesos recursivos de Flower y Hayes (1981): planificación de la escritura, textualización o la producción escrita del texto y la revisión. Las "estrategias de regulación" tienen como objetivo conocer cómo el estudiante autorregula la escritura (qué estrategias cognitivas y metacognitivas utiliza mientras escribe). Las "concepciones sobre la escritura" pretenden dar cuenta de las creencias que tienen los estudiantes a partir de sus experiencias mientras escriben, y la variable de "autoeficacia" se relaciona con la autopercepción que tienen los estudiantes respecto de sus capacidades para llevar a cabo la tarea de escritura. Los resultados obtenidos por Difabio $(2012$; 2013) y Álvarez y Difabio (2017) dan cuenta de que el instrumento tiene una adecuada calidad psicométrica, por lo que logra medir apropiadamente las percepciones de los sujetos hacia la escritura académica en las variables consideradas.

En relación con esto, se debe señalar que hay pocas investigaciones que aborden las concepciones de la escritura, y las que existen, proceden de distintas perspectivas teóricas. Villalón y Mateos (2009) clasifican los estudios sobre percepción de escritura en tres enfoques: fenomenográfico, metacognitivo y en la teoría social del aprendizaje.

En el enfoque fenomenográfico se sitúan cuatro trabajos relevantes: (1) Hounsell (1984), referido a las concepciones sobre el género ensayo en estudiantes de Historia, donde encuentra tres percepciones diferentes: argumento, entendido como la evidencia que sustenta una postura sobre un tema, referencias a los 
datos que apoyan el argumento y la expresión de ideas y opiniones; (2) Campbell, Smith y Brooker (1998), mediante entrevistas a estudiantes universitarios sobre cómo abordan el ensayo, obtienen una correlación entre, por un lado, ensayos más elaborados y percepciones constructivistas y, por otro, ensayos simplistas y percepciones reproductivas; (3) Lavelle (1993) señala que, según la intención del alumno hacia la escritura, elige distintas estrategias que impactan en su escritura; y (4) Lavelle y Bushrow (2007), en el contexto del posgrado, obtienen percepciones intuitivas, elaborativas y científicas por parte de los estudiantes.

En el enfoque metacognitivo se encuentran los aportes de Scardamalia y Bereiter (1992), quienes plantean dos modelos del proceso de escritura: decir el conocimiento y transformar el conocimiento. El primero se refiere a un escritor inmaduro, que transfiere sus ideas de manera lineal, tal como se le van ocurriendo con un procesamiento superficial. El segundo corresponde a un escritor experto, que toma el modelo anterior, pero va más allá, puesto que logra una interacción entre el contenido de lo que expresa y las estrategias que utiliza para hacerlo.

En el enfoque de la teoría social del aprendizaje, los especialistas investigan el efecto de las percepciones de los alumnos sobre la escritura en la producción de sus textos; por ejemplo, Villalón (2010) encuentra que los alumnos universitarios muestran conceptualizaciones más elaboradas que los alumnos de enseñanza secundaria; no obstante, ambos grupos están lejos de lograr una concepción epistémica de la escritura o, en palabras de Scardamalia y Bereiter, de "transformar el conocimiento", es decir, hacer modificaciones y adecuaciones de su composición acorde con los objetivos que guían la escritura.

\section{Método}

En este artículo se presenta un estudio no experimental, cuantitativo, con un diseño transeccional exploratorio (es decir, se recolectaron los datos en un solo momento).

\section{Participantes}

En este estudio participaron 52 estudiantes de una universidad chilena, 32 de Ingeniería Civil Electrónica y 20 de Traducción. Los alumnos de Ingeniería se encontraban cursando segundo, tercer y cuarto año de la carrera, y los de traducción, segundo año.

En el momento de la aplicación del cuestionario, los dos grupos de estudiantes, por separado, cursaban una asignatura semestral de escritura académica.

\section{Instrumento}

El cuestionario corresponde a una escala Likert de 5 puntos (1: totalmente en desacuerdo; 2: en desacuerdo; 3: indiferente; 4: de acuerdo, y 5: muy de acuerdo) y está compuesto por 36 ítems, 22 positivos o favorables, y 14 reversos o negativos. Este instrumento se aplicó en papel a los dos grupos de estudiantes.

El cuestionario se elaboró teniendo en cuenta la escala diseñada por Difabio (2012). Diecinueve de las preguntas referidas al proceso general de escritura fueron tomadas del inventario propuesto por Difabio (2012); seis de las preguntas se tomaron de Rodríguez y García (2015) sobre las diferentes etapas del proceso de escribir y once se crearon ad hoc, según los objetivos de la investigación.

El cuestionario contempla cinco dimensiones: planificación, textualización, revisión, postergación del inicio de la escritura y actitudes hacia la escritura. A continuación, se define operacionalmente cada una de ellas.

Planificación. Esta etapa se asume como un proceso de reflexión, previo a la escritura. En términos generales, suele ser una fase omitida o innecesaria para escritores inexpertos.

El resultado de no considerar la planificación antes de comenzar a escribir conduce a un texto desorganizado, no adaptado a lo que para el autor pueden ser las expectativas del lector. 
En el cuestionario hay siete ítems que evalúan esta dimensión:

2. Dedico un tiempo a pensar sobre el tema que voy a escribir.

7. Tengo claridad respecto del lector de mi texto.

9. Comienzo a escribir con algún plan de escritura (esquema con las ideas principales).

10. Antes de comenzar a escribir tengo claridad respecto de la estructura del texto que tengo que elaborar.

21. Dedico un espacio suficiente a la recopilación de información que me servirá para elaborar mi escrito. 30. Tengo claridad respecto de los objetivos que guían mi escritura.

32. Mi escritura simplemente "ocurre" sin mayor planificación.

Textualización. Es el momento en que se plasma, en el papel u otro soporte, el esquema de ideas elaborado en la etapa de planificación de la escritura.

En el cuestionario se presentan nueve ítems en esta dimensión:

1. Me resulta difícil presentar mi propia voz dentro del texto, porque mi escritura se apega a lo que dicen otros autores.

5. Me resulta difícil mantener un lenguaje objetivo y formal mientras escribo.

6. Me cuesta ser consciente de que cada párrafo debe plantear una idea principal.

14. Trato de escribir oraciones efectivas de transición desde una idea a otra.

18. Me cuesta presentar distintas posturas sobre el tema que escribo.

19. Es difícil para mí conectar los distintos párrafos.

23. Mientras escribo un texto académico, voy desarrollando mis ideas.

29. Trato de encontrar una idea principal clara que explica el tema de mi escrito.

33. Tengo conocimientos respecto de las secuencias textuales que debe presentar un texto académico.

Revisión. La etapa de revisión textual es relevante en el proceso de redacción. Según Rodríguez y García (2015), esta puede realizarse en dos niveles: local (durante la escritura, por ejemplo, una idea o un párrafo) y global (corresponde a la revisión del texto completo).

En este sentido, en el cuestionario esta dimensión se evalúa mediante nueve ítems:
3. Reviso el borrador para hacerlo más organizado.

8. Cuando he escrito un texto largo, me esfuerzo por encontrar y corregir todos mis errores gramaticales y de estilo.

12. Cuando termino el texto, no tengo tiempo para revisarlo.

17. No reviso lo que he escrito, porque simplemente no puedo ver mis errores.

20. Suelo revisar mi escrito varias veces antes de considerarlo terminado.

22. Con frecuencia, mi primer borrador es mi producto final.

24. Después de escribir, reviso mi texto para observar si tiene faltas de ortografía.

28. Cuando termino de escribir el texto, dedico un tiempo a revisarlo.

31. Después de escribir mi texto, reviso si expresé claramente las ideas; de lo contrario, las modifico.

Postergación del inicio de la escritura. De acuerdo con Carlino (2004), una de las dificultades que tienen los estudiantes cuando escriben se debe a que postergan el momento de realizar esta actividad. Difabio (2012) señala que esto ocurre porque el escritor tiene miedo al fracaso, ansiedad, falta de autoconfianza, etc. Esta postergación lleva a una producción de textos en los que el escritor plasma sus ideas en el mismo orden en que le fueron surgiendo (prosa basada en el autor).

En el cuestionario se consideran tres ítems para evaluar esta dimensión:

\section{Me cuesta empezar a escribir.}

13. Me preocupo tanto por mis dificultades para la escritura que se me hace arduo empezar a escribir.

26. Pospongo la tarea de escritura hasta el último momento.

Actitudes hacia la escritura. Corresponde a las creencias que tienen los estudiantes respecto de sus capacidades para llevar a cabo la tarea de escribir.

Esta dimensión se mide en el cuestionario a través de ocho ítems:
4. Puedo empezar a escribir con facilidad.
15. El proceso de escritura me resulta estresante.
16. Detesto escribir.
25. La escritura me hace sentir bien.
27. Tengo facilidad para escribir. 
34. Me gusta que las tareas de escritura que me asignan, tengan pautas claras, con todos los detalles.

35. Lo más importante en la escritura es respetar las reglas de gramática, puntuación y organización.

36. Si estudiara gramática y puntuación, mi escritura mejoraría mucho.

Por otro lado, debe señalarse que la autora cumplió todas las obligaciones legales aplicables en su país, lo que incluye la aprobación de los comités institucionales de vigilancia, la información a los participantes de los detalles y ausencia de riesgos por participar del estudio, y se aseguró el consentimiento firmado de los estudiantes.

Para el análisis de los datos, se utilizó el software IBM SPSS Statistics V22.0.

\section{Resultados}

De acuerdo con el objetivo del estudio, se presentan cinco tablas, con los resultados generales y diferenciados por grupos de estudiantes en las distintas dimensiones del cuestionario. En la Tabla 1 se dan a conocer las respuestas de los estudiantes en relación con la primera dimensión del cuestionario, la "planificación”.

En términos generales, se observa en la Tabla 1 que los alumnos de Ingeniería y de Traducción tienen opiniones similares respecto de la planificación en la escritura de textos académicos. En la pregunta 2, el 78,8 \% (59,6\%, "De acuerdo", y 19,2 \%, "Muy de acuerdo") de los estudiantes reconocen que

Tabla 1 Dimensión "Planificación"

\begin{tabular}{|c|c|c|c|c|c|c|c|c|}
\hline $\begin{array}{l}\text { Número de } \\
\text { pregunta }\end{array}$ & Área & $\begin{array}{c}\text { Totalmente } \\
\text { en } \\
\text { desacuerdo } \\
(\%)\end{array}$ & $\begin{array}{c}\text { En } \\
\text { desacuerdo } \\
(\%)\end{array}$ & $\begin{array}{c}\text { Indiferente } \\
(\%)\end{array}$ & $\begin{array}{c}\text { De acuerdo } \\
(\%)\end{array}$ & $\begin{array}{c}\text { Muy de } \\
\text { acuerdo } \\
(\%)\end{array}$ & Estadígrafo* & $\begin{array}{c}\text { Valor } \\
\mathbf{p}\end{array}$ \\
\hline \multirow[t]{3}{*}{2} & Total & 0,0 & 11,5 & 9,6 & 59,6 & 19,2 & 3,53 & 0,3379 \\
\hline & Ingeniería & 0,0 & 15,6 & 12,5 & 59,4 & 12,5 & & \\
\hline & Traducción & 0,0 & 5,0 & 5,0 & 60,0 & 30,0 & & \\
\hline \multirow[t]{3}{*}{7} & Total & 1,9 & 28,8 & 26,9 & 40,4 & 1,9 & 4,84 & 0,2685 \\
\hline & Ingeniería & 3,1 & 37,5 & 21,9 & 34,4 & 3,1 & & \\
\hline & Traducción & 0,0 & 15,0 & 35,0 & 50,0 & 0,0 & & \\
\hline \multirow[t]{3}{*}{9} & Total & 11,5 & 42,3 & 9,6 & 30,8 & 5,8 & 6,53 & 0,1459 \\
\hline & Ingeniería & 12,5 & 37,5 & 12,5 & 37,5 & 0,0 & & \\
\hline & Traducción & 10,0 & 50,0 & 5,0 & 20,0 & 15,0 & & \\
\hline \multirow[t]{3}{*}{10} & Total & 1,9 & 23,1 & 23,1 & 44,2 & 7,7 & 4,15 & 0,3962 \\
\hline & Ingeniería & 3,1 & 18,8 & 25,0 & 50,0 & 3,1 & & \\
\hline & Traducción & 0,0 & 30,0 & 20,0 & 35,0 & 15,0 & & \\
\hline \multirow[t]{3}{*}{21} & Total & 1,9 & 26,9 & 19,2 & 38,5 & 13,5 & 1,67 & 0,9014 \\
\hline & Ingeniería & 3,1 & 25,0 & 21,9 & 34,4 & 15,6 & & \\
\hline & Traducción & 0,0 & 30,0 & 15,0 & 45,0 & 10,0 & & \\
\hline \multirow[t]{3}{*}{30} & Total & 0,0 & 34,6 & 23,1 & 30,8 & 11,5 & 0,85 & 0,8751 \\
\hline & Ingeniería & 0,0 & 31,3 & 21,9 & 34,4 & 12,5 & & \\
\hline & Traducción & 0,0 & 40,0 & 25,0 & 25,0 & 10,0 & & \\
\hline \multirow[t]{3}{*}{32} & Total & 5,8 & 32,7 & 9,6 & 42,3 & 9,6 & 2,84 & 0,5937 \\
\hline & Ingeniería & 3,1 & 37,5 & 6,3 & 43,8 & 9,4 & & \\
\hline & Traducción & 10,0 & 25,0 & 15,0 & 40,0 & 10,0 & & \\
\hline
\end{tabular}

* Estadígrafo asociado a la obtención del test exacto de Fisher. 
dedican un tiempo a pensar sobre el tema que van a escribir. Asimismo, en el ítem 7, el 34,4\% de los alumnos de Ingeniería está de acuerdo en que tienen claridad respecto del lector del texto al que le van a escribir. Estos resultados coinciden con los de Errázuriz et al. (2015), donde la mayoría de los alumnos de pedagogía reconocen que no tienen en cuenta al lector, pues dan por hecho que si ellos entienden sus ideas, el lector también lo hará.

En el ítem 9, el 42,3\% de los alumnos está en desacuerdo con la idea de que su escritura comience con un esquema previo, lo cual se relaciona con el resultado de las preguntas 30 y 32 , en las que, respectivamente, el 34,6\% señala que no tiene claridad acerca de los objetivos que guían su escritura, y el 51,9 \% (42,3\%, "De acuerdo" y 9,6 \%, "Muy de acuerdo") declara que su escritura "ocurre" sin mayor planificación. En la pregunta 21 , un $52 \%$ de la muestra (38,5 \% "De acuerdo" y 13,5 \% "Muy de acuerdo") declara que dedican un espacio para recopilar información que servirá de base para elaborar sus textos.

Dado que se observan tendencias similares en las respuestas de ambos grupos, la prueba estadística de Fisher no demuestra diferencias significativas entre ellos. Según Carlino (2004) y Rodríguez y García (2015), se necesita que los alumnos puedan ser conscientes de la relevancia que tiene la planificación en la producción efectiva de un escrito.

En la Tabla 2 se aprecia una dispersión en las respuestas de la muestra en la dimensión de "textualización", puesto que en el ítem 1, el 36,5\% afirma que no le es difícil presentar su propia voz dentro del texto, mientras que el $32,7 \%$ reconoce que es un problema para ellos desapegarse de las ideas expuestas por otros autores en los cuales basan sus escritos, lo que resulta más problemático en el grupo de Ingeniería.

En el ítem 5, el 34,6 \% (la suma de "De acuerdo" y "Muy de acuerdo") de la muestra señala que le resulta complejo mantener un lenguaje objetivo y formal mientras escribe. Esto demuestra que escribir implica una serie de cuestiones, entre ellas, organizativas, textuales, léxicas, etc., y esto se relaciona con la inmersión de los estudiantes en el lenguaje propio de las disciplinas y sus géneros (Errázuriz et al., 2015).

Los estudiantes de ambos grupos coinciden en sus respuestas a las preguntas 6, 14 y 18; esto significa que, respectivamente, les resulta difícil ser conscientes de que cada párrafo debe plantear una idea principal; sin embargo, el 55,8 \% de los alumnos intenta escribir oraciones adecuadas, que les permita avanzar en el planteamiento de las ideas (pregunta 14); por último, el 46,1 \% (42,3\%, "De acuerdo", y 3,8 \%, "Muy de acuerdo" en pregunta 18) reconoce que le cuesta plantear distintas posturas sobre el tema que escribe. Esto indica que las habilidades argumentativa y contraaargumentativa son dos de las más complejas en el contexto de la escritura académica (Errázuriz et al., 2015).

En la pregunta 19 se observan divergencias entre ambos grupos: mientras el 53,1\% de los alumnos de Ingeniería evidencia dificultades para conectar los distintos párrafos en un texto, el $60 \%$ de los estudiantes de Traducción señala que para ellos esto no representa un problema. Estas diferencias son significativas a nivel estadístico entre ambos grupos, con un valor $p=0,0141$. Una de las explicaciones es que ello puede deberse a la frecuencia de la práctica de escritura en las Ciencias Humanas por sobre las Ciencias de Ingeniería, que si bien producen textos, la mayoría de sus escritos corresponden a respuestas breves dirigidas (resolución de problemas, cálculos matemáticos, etc.), cuyas soluciones evidencian la integración de la competencia lectora y escrita del estudiante.

En relación con la pregunta 23, el 65,4\% de la muestra (59,6 \%, "De acuerdo" y 5,8 \%, "Muy de acuerdo") va desarrollando sus ideas mientras escribe el texto, lo que se vincula con los resultados señalados en la Tabla 1 en cuanto a que los alumnos comienzan a escribir sin un esquema previo.

En el ítem 29, un 71,1\% (59,6\% “De acuerdo" y $11,5 \%$ "Muy de acuerdo") de la muestra intenta encontrar una idea principal que logre dejar claro de 
Tabla 2 Dimensión "Textualización”

\begin{tabular}{|c|c|c|c|c|c|c|c|c|}
\hline $\begin{array}{l}\text { Número } \\
\text { de } \\
\text { pregunta }\end{array}$ & Área & $\begin{array}{l}\text { Totalmente en } \\
\text { desacuerdo (\%) }\end{array}$ & $\begin{array}{l}\text { En } \\
\text { desacuerdo } \\
\text { (\%) }\end{array}$ & $\begin{array}{l}\text { Indiferente } \\
(\%)\end{array}$ & $\begin{array}{l}\text { De acuerdo } \\
\text { (\%) }\end{array}$ & $\begin{array}{l}\text { Muy de } \\
\text { acuerdo } \\
\text { (\%) }\end{array}$ & Estadígrafo & Valor $p$ \\
\hline \multirow[t]{3}{*}{1} & Total & 13,5 & 36,5 & 17,3 & 32,7 & 0,0 & 1,88 & 0,6261 \\
\hline & Ingeniería & 12,5 & 37,5 & 12,5 & 37,5 & 0,0 & & \\
\hline & Traducción & 15,0 & 35,0 & 25,0 & 25,0 & 0,0 & & \\
\hline \multirow[t]{3}{*}{5} & Total & 13,5 & 38,5 & 13,5 & 30,8 & 3,8 & 4,62 & 0,3281 \\
\hline & Ingeniería & 12,5 & 34,4 & 9,4 & 40,6 & 3,1 & & \\
\hline & Traducción & 15,0 & 45,0 & 20,0 & 15,0 & 5,0 & & \\
\hline \multirow[t]{3}{*}{6} & Total & 0,0 & 26,9 & 15,4 & 48,1 & 9,6 & 3,08 & 0,4115 \\
\hline & Ingeniería & 0,0 & 25,0 & 9,4 & 53,1 & 12,5 & & \\
\hline & Traducción & 0,0 & 30,0 & 25,0 & 40,0 & 5,0 & & \\
\hline \multirow[t]{3}{*}{14} & Total & 0,0 & 17,3 & 23,1 & 55,8 & 3,8 & 4,88 & 0,1456 \\
\hline & Ingeniería & 0,0 & 25,0 & 21,9 & 46,9 & 6,3 & & \\
\hline & Traducción & 0,0 & 5,0 & 25,0 & 70,0 & 0,0 & & \\
\hline \multirow[t]{3}{*}{18} & Total & 7,7 & 21,2 & 25,0 & 42,3 & 3,8 & 1,88 & 0,7901 \\
\hline & Ingeniería & 9,4 & 18,8 & 21,9 & 43,8 & 6,3 & & \\
\hline & Traducción & 5,0 & 25,0 & 30,0 & 40,0 & 0,0 & & \\
\hline \multirow[t]{3}{*}{$19^{*}$} & Total & 1,9 & 38,5 & 17,3 & 38,5 & 3,8 & 10,60 & 0,0141 \\
\hline & Ingeniería & 0,0 & 25,0 & 18,8 & 53,1 & 3,1 & & \\
\hline & Traducción & 5,0 & 60,0 & 15,0 & 15,0 & 5,0 & & \\
\hline \multirow[t]{3}{*}{23} & Total & 1,9 & 17,3 & 15,4 & 59,6 & 5,8 & 2,42 & 0,7841 \\
\hline & Ingeniería & 0,0 & 18,8 & 12,5 & 62,5 & 6,3 & & \\
\hline & Traducción & 5,0 & 15,0 & 20,0 & 55,0 & 5,0 & & \\
\hline \multirow[t]{3}{*}{29} & Total & 0,0 & 17,3 & 11,5 & 59,6 & 11,5 & 3,89 & 0,2755 \\
\hline & Ingeniería & 0,0 & 25,0 & 12,5 & 53,1 & 9,4 & & \\
\hline & Traducción & 0,0 & 5,0 & 10,0 & 70,0 & 15,0 & & \\
\hline \multirow[t]{3}{*}{$33^{* * *}$} & Total & 9,6 & 38,5 & 15,4 & 36,5 & 0,0 & 23,00 & $<0,0001$ \\
\hline & Ingeniería & 15,6 & 56,3 & 15,6 & 12,5 & 0,0 & & \\
\hline & Traducción & 0,0 & 10,0 & 15,0 & 75,0 & 0,0 & & \\
\hline
\end{tabular}

${ }^{*}$ valor $p<0,05$

${ }^{* *}$ valor $p<0,01$

qué trata su escrito. Constituye una tarea compleja, donde el proceso de planificación de la escritura cumple un rol fundamental.

En la pregunta 33 se observa que el dominio de las secuencias textuales resulta problemático para los estudiantes de Ingeniería; así lo reconoce un 71,9\% (si se suman los porcentajes de "Totalmente en desacuerdo" y "En desacuerdo"), mientras que los alumnos de Traducción reconocen, en un $75 \%$, que tienen algún tipo de formación o conocimiento respecto de las secuencias textuales (por ejemplo, la narración, la descripción, etc.).

Estos resultados demuestran la necesidad de que en las distintas carreras se dicten asignaturas relacionadas con el proceso de escritura en la universidad (Carlino, 2003; Ortiz, 2011). Al respecto, la carrera de Ingeniería Civil Electrónica, presentada en este estudio, solo considera un curso semestral de 
escritura académica en toda su formación (en el tercer año). En la carrera de Traducción, el curso de escritura académica se ubica en el segundo año de formación y luego los estudiantes tienen otra asignatura semestral que favorece sus prácticas escritas y orales en contextos académicos.

La Tabla 3 presenta las respuestas de los sujetos en relación con los ítems que miden la tercera dimensión del cuestionario, que corresponde a la "revisión" del texto escrito. Los resultados del ítem 3 evidencian diferencias notorias entre los dos grupos, con un valor $p=0,0018$. Así el $70 \%$ de los estudiantes de Traducción (si se suman los porcentajes de las respuestas "De acuerdo" y "Muy de acuerdo") reconocen que revisan el borrador de su escrito para hacerlo más organizado; a diferencia de los alumnos de Ingeniería, quienes consideran, en menor medida, el proceso de revisión en la etapa de escritura de sus textos.

Tabla 3 Dimensión "Revisión”

\begin{tabular}{|c|c|c|c|c|c|c|c|c|}
\hline $\begin{array}{l}\text { Número } \\
\text { de } \\
\text { pregunta }\end{array}$ & Área & $\begin{array}{c}\text { Totalmente en } \\
\text { desacuerdo } \\
(\%)\end{array}$ & $\begin{array}{c}\text { En } \\
\text { desacuerdo } \\
(\%)\end{array}$ & $\begin{array}{c}\text { Indiferente } \\
(\%)\end{array}$ & $\begin{array}{c}\text { De acuerdo } \\
(\%)\end{array}$ & $\begin{array}{c}\text { Muy de } \\
\text { acuerdo } \\
(\%)\end{array}$ & Estadígrafo & Valor $p$ \\
\hline \multirow[t]{3}{*}{$3^{*}$} & Total & 7,7 & 19,2 & 19,2 & 38,5 & 15,4 & 15,39 & 0,0018 \\
\hline & Ingeniería & 12,5 & 31,3 & 12,5 & 37,5 & 6,3 & & \\
\hline & Traducción & 0,0 & 0,0 & 30,0 & 40,0 & 30,0 & & \\
\hline \multirow[t]{3}{*}{8} & Total & 3,8 & 25,0 & 3,8 & 42,3 & 25,0 & 7,90 & 0,0587 \\
\hline & Ingeniería & 6,3 & 34,4 & 6,3 & 37,5 & 15,6 & & \\
\hline & Traducción & 0,0 & 10,0 & 0,0 & 50,0 & 40,0 & & \\
\hline \multirow[t]{3}{*}{12} & Total & 5,8 & 46,2 & 21,2 & 19,2 & 7,7 & 3,43 & 0,5099 \\
\hline & Ingeniería & 6,3 & 40,6 & 18,8 & 21,9 & 12,5 & & \\
\hline & Traducción & 5,0 & 55,0 & 25,0 & 15,0 & 0,0 & & \\
\hline \multirow[t]{3}{*}{17} & Total & 17,3 & 50,0 & 15,4 & 15,4 & 1,9 & 2,33 & 0,7626 \\
\hline & Ingeniería & 12,5 & 50,0 & 15,6 & 18,8 & 3,1 & & \\
\hline & Traducción & 25,0 & 50,0 & 15,0 & 10,0 & 0,0 & & \\
\hline \multirow[t]{3}{*}{20} & Total & 3,8 & 34,6 & 17,3 & 28,8 & 15,4 & 5,25 & 0,2540 \\
\hline & Ingeniería & 6,3 & 40,6 & 15,6 & 18,8 & 18,8 & & \\
\hline & Traducción & 0,0 & 25,0 & 20,0 & 45,0 & 10,0 & & \\
\hline \multirow[t]{3}{*}{22} & Total & 7,7 & 46,2 & 9,6 & 32,7 & 3,8 & 5,21 & 0,2320 \\
\hline & Ingeniería & 3,1 & 40,6 & 12,5 & 40,6 & 3,1 & & \\
\hline & Traducción & 15,0 & 55,0 & 5,0 & 20,0 & 5,0 & & \\
\hline \multirow[t]{3}{*}{24} & Total & 0,0 & 5,8 & 3,8 & 44,2 & 46,2 & 3,19 & 0,3376 \\
\hline & Ingeniería & 0,0 & 9,4 & 6,3 & 37,5 & 46,9 & & \\
\hline & Traducción & 0,0 & 0,0 & 0,0 & 55,0 & 45,0 & & \\
\hline \multirow[t]{3}{*}{$28^{*}$} & Total & 0,0 & 23,1 & 13,5 & 50,0 & 13,5 & 12,14 & 0,0052 \\
\hline & Ingeniería & 0,0 & 34,4 & 15,6 & 31,3 & 18,8 & & \\
\hline & Traducción & 0,0 & 5,0 & 10,0 & 80,0 & 5,0 & & \\
\hline \multirow[t]{3}{*}{$31^{* *}$} & Total & 0,0 & 23,1 & 7,7 & 51,9 & 17,3 & 7,57 & 0,0467 \\
\hline & Ingeniería & 0,0 & 34,4 & 3,1 & 46,9 & 15,6 & & \\
\hline & Traducción & 0,0 & 5,0 & 15,0 & 60,0 & 20,0 & & \\
\hline
\end{tabular}

${ }^{*}$ valor $\mathrm{p}<0,01$

${ }^{* *}$ valor $\mathrm{p}<0,05$ 
En la pregunta 8 se observa una leve significancia estadística entre ambos grupos, lo cual indica que los alumnos de Traducción se preocupan más de corregir sus errores gramaticales y de estilo, luego de haber escrito un texto, que los estudiantes de Ingeniería. En el reactivo 12, la muestra reconoce que después de escribir un texto, deja un tiempo para revisarlo, por lo que un 46,2 \% marcó la opción "En desacuerdo" frente a este ítem negativo: "Cuando termino el texto, no tengo tiempo para revisarlo".

En relación con las respuestas al ítem 17, expresado de forma negativa ("17. No reviso lo que he escrito, porque simplemente no puedo ver mis errores"), es preciso señalar que los sujetos son conscientes de que cometen errores en su escritura, por lo que dedican un tiempo a revisarla. No obstante, cabría preguntarse cuál es el nivel de competencia que presentan estos alumnos para poder "ver" y corregir sus errores, principalmente, en Ingeniería, donde solo tienen una asignatura semestral de escritura académica en toda su formación universitaria.

Las respuestas de la pregunta 20 revelan que el 40,6 \% de los alumnos de Ingeniería no acostumbran a revisar sus escritos varias veces antes de considerarlos terminados; no sucede esto con los estudiantes de Traducción, quienes reconocen, en un $55 \%$ (45\% "De acuerdo" y $10 \%$ "Muy de acuerdo"), que otorgan un espacio relevante a esta actividad. Pese a ello, en el ítem 22, el 46,2 \% de la muestra está en desacuerdo con la idea de que su primer borrador se constituya en su texto final.

En la pregunta 24 se observan altos porcentajes en las categorías "De acuerdo" y "Muy de acuerdo" en ambos grupos; esto significa que los alumnos reconocen que luego de escribir su texto dejan un espacio para revisar las faltas ortográficas, lo cual se relaciona con lo planteado por Carlino (2004): que la revisión, generalmente, se queda en el nivel superficial de la corrección.

En el ítem 28 se aprecian diferencias estadísticamente significativas entre los grupos, con un valor $p=0,0052$, debido a que solo el 50,1\% de los alumnos de Ingeniería expresan que dedican un tiempo a la revisión del texto que han escrito, mientras que el $85 \%$ de los estudiantes de Traducción reconocen hacerlo.

Respecto de la pregunta 31, sobre si después de escribir su texto, los alumnos revisan si expresaron claramente sus ideas, o de lo contrario las modifican, se observa en la Tabla 3 que el $80 \%$ de los de Traducción reconocen que hacen cambios en sus ideas para expresarlas con claridad. Si bien asimismo se aprecia un porcentaje elevado en los alumnos de Ingeniería, este es notablemente inferior al de Traducción, lo que se corrobora con el valor significativo de $p=0,0467$. Dichos resultados concuerdan con lo señalado por Carlino (2004) y Errázuriz et al. (2015) respecto a que los estudiantes tienden a conservar sus ideas, y si realizan cambios, estos solo son superficiales.

En la Tabla 4 se muestran las respuestas correspondientes a los ítems de la cuarta dimensión, "Postergación del inicio de la escritura". En esta dimensión se destaca el ítem 13, que demuestra diferencias significativas desde un punto de vista estadístico entre ambos grupos, con un valor $p=0,0477$. Esto significa que los alumnos de Traducción evitan posponer la escritura para el último momento, mientras que las respuestas en Ingeniería son más dispersas, puesto que el mismo porcentaje de estudiantes está en "Desacuerdo", "De acuerdo" e "Indiferente" con esta afirmación. Dejar la escritura para el final es señal de inseguridad respecto de lo que se espera del texto, o bien el resultado de una tarea vaga, sin pautas definidas. Para Carlino (2004), cuando se posterga la escritura, se obtiene una prosa basada en el autor, es decir, las ideas se plasman como fueron surgiendo en la mente del que escribe. Esto se reafirma con la percepción del ítem 11, donde el 55,8 \% (38,5 \% "De acuerdo" y 17,3 \% "Muy de acuerdo") de la muestra reconoce que les cuesta empezar a escribir, y por lo mismo, el 53,9\% (38,5 \% "De acuerdo" y 15,4\% "Muy de acuerdo") de los estudiantes posponen la tarea de escribir hasta el último momento. 
Tabla 4 Dimensión "Postergación del inicio de la escritura"

\begin{tabular}{|c|c|c|c|c|c|c|c|c|}
\hline $\begin{array}{l}\text { Número de } \\
\text { pregunta }\end{array}$ & Área & $\begin{array}{l}\text { Totalmente } \\
\text { en } \\
\text { desacuerdo } \\
(\%)\end{array}$ & $\begin{array}{l}\text { En } \\
\text { desacuerdo } \\
(\%)\end{array}$ & $\begin{array}{l}\text { Indiferente } \\
(\%)\end{array}$ & $\begin{array}{l}\text { De acuerdo } \\
(\%)\end{array}$ & $\begin{array}{l}\text { Muy de } \\
\text { acuerdo } \\
(\%)\end{array}$ & Estadígrafo & Valor $p$ \\
\hline \multirow[t]{3}{*}{11} & Total & 1,9 & 28,8 & 13,5 & 38,5 & 17,3 & 2,98 & 0,6054 \\
\hline & Ingeniería & 0,0 & 34,4 & 12,5 & 34,4 & 18,8 & & \\
\hline & Traducción & 5,0 & 20,0 & 15,0 & 45,0 & 15,0 & & \\
\hline \multirow[t]{3}{*}{$13 *$} & Total & 9,6 & 28,8 & 28,8 & 23,1 & 9,6 & 9,12 & 0,0477 \\
\hline & Ingeniería & 12,5 & 28,1 & 31,3 & 28,1 & 0,0 & & \\
\hline & Traducción & 5,0 & 30,0 & 25,0 & 15,0 & 25,0 & & \\
\hline \multirow[t]{3}{*}{26} & Total & 1,9 & 30,8 & 13,5 & 38,5 & 15,4 & 2,52 & 0,7165 \\
\hline & Ingeniería & 3,1 & 28,1 & 9,4 & 40,6 & 18,8 & & \\
\hline & Traducción & 0,0 & 35,0 & 20,0 & 35,0 & 10,0 & & \\
\hline
\end{tabular}

${ }^{*}$ valor $\mathrm{p}<0,05$

La Tabla 5 da a conocer el resultado obtenido por la muestra en la quinta dimensión, "Actitudes hacia la escritura". En cuanto al ítem 4, se puede observar que el 38,5\% de los estudiantes está en desacuerdo con la afirmación "Puedo empezar a escribir con facilidad". Sin embargo, esta dificultad se manifiesta más claramente en el grupo de Ingeniería, con un $46,9 \%$, lo cual se puede explicar por el poco hábito que se tiene en la escritura de textos, a diferencia de los estudiantes de la carrera de Traducción, donde la producción de textos extensos es una práctica frecuente.

En relación con la pregunta 15 , se aprecian opiniones diversas entre los estudiantes. No obstante, se puede señalar, a partir de los datos, que para el 40,6 \% de los alumnos de Ingeniería (25\%, "De acuerdo", y 15,6\%, "Muy de acuerdo"), escribir un texto les resulta estresante. Pese a ello, un reducido porcentaje detesta escribir; así lo confirman sus respuestas al ítem 16.

En el ítem 25, el $50 \%$ de los estudiantes de Traducción declaran que la escritura los hace sentirse bien, mientras que los de Ingeniería se inclinan mayoritariamente por la opción "Indiferente".

Los resultados presentados en la pregunta 27 son interesantes, puesto que un 44,3\% (sumando "De acuerdo" y "Muy de acuerdo") reconoce que tienen facilidad para escribir, por lo que se debería impulsar esta habilidad a través de la alfabetización académica en las distintas disciplinas.

Una dificultad que enfrentan los estudiantes cuando deben producir un texto se debe a la falta de lineamientos claros respecto de lo que se espera de su escritura (Figueras y Puig, 2013; Martínez, 2011). En este contexto, en el ítem 34, el 67,3 \% (30,8\%, "De acuerdo" y 36,5 \%, "Muy de acuerdo") de la muestra prefiere que las tareas de escritura que se le asignan tengan pautas detalladas respecto de lo que se espera en su producción.

En cuanto al ítem 35, el 73,1 \% de la muestra (50\%, "De acuerdo", y 23,1\%, "Muy de acuerdo") considera que lo más importante en la escritura es respetar las reglas gramaticales, de puntuación y de organización textual. Este porcentaje representa más claramente a los alumnos de Ingeniería, pues el 81,3\% de ellos comparte la idea presentada anteriormente, ya que los de Traducción representan al $60 \%$ del alumnado. Dicha percepción podría explicarse porque los alumnos de Traducción comprenden que el proceso de escritura incluye otros aspectos, además de los puramente gramaticales, como: coherencia, cohesión, desarrollo de ideas, etc. Sin embargo, en el ítem 
Tabla 5 Dimensión "Actitudes hacia la escritura"

\begin{tabular}{|c|c|c|c|c|c|c|c|c|}
\hline $\begin{array}{l}\text { Número } \\
\text { de } \\
\text { pregunta }\end{array}$ & Área & $\begin{array}{l}\text { Totalmente } \\
\text { en } \\
\text { desacuerdo } \\
(\%)\end{array}$ & $\begin{array}{l}\text { En } \\
\text { desacuerdo } \\
(\%)\end{array}$ & $\begin{array}{l}\text { Indiferente } \\
(\%)\end{array}$ & $\begin{array}{l}\text { De } \\
\text { acuerdo } \\
(\%)\end{array}$ & $\begin{array}{l}\text { Muy de } \\
\text { acuerdo } \\
(\%)\end{array}$ & Estadígrafo & Valor $p$ \\
\hline \multirow[t]{3}{*}{4} & Total & 11,5 & 38,5 & 23,1 & 25,0 & 1,9 & 4,77 & 0,2820 \\
\hline & Ingeniería & 6,3 & 46,9 & 18,8 & 25,0 & 3,1 & & \\
\hline & Traducción & 20,0 & 25,0 & 30,0 & 25,0 & 0,0 & & \\
\hline \multirow[t]{3}{*}{15} & Total & 9,6 & 25,0 & 26,9 & 21,2 & 17,3 & 2,18 & 0,7299 \\
\hline & Ingeniería & 12,5 & 25,0 & 21,9 & 25,0 & 15,6 & & \\
\hline & Traducción & 5,0 & 25,0 & 35,0 & 15,0 & 20,0 & & \\
\hline \multirow[t]{3}{*}{16} & Total & 34,6 & 38,5 & 13,5 & 9,6 & 3,8 & 2,78 & 0,6151 \\
\hline & Ingeniería & 28,1 & 37,5 & 15,6 & 12,5 & 6,3 & & \\
\hline & Traducción & 45,0 & 40,0 & 10,0 & 5,0 & 0,0 & & \\
\hline \multirow[t]{3}{*}{25} & Total & 5,8 & 17,3 & 38,5 & 26,9 & 11,5 & 6,41 & 0,1549 \\
\hline & Ingeniería & 9,4 & 18,8 & 40,6 & 28,1 & 3,1 & & \\
\hline & Traducción & 0,0 & 15,0 & 35,0 & 25,0 & 25,0 & & \\
\hline \multirow[t]{3}{*}{27} & Total & 17,3 & 23,1 & 15,4 & 38,5 & 5,8 & 0,99 & 0,9587 \\
\hline & Ingeniería & 18,8 & 25,0 & 12,5 & 37,5 & 6,3 & & \\
\hline & Traducción & 15,0 & 20,0 & 20,0 & 40,0 & 5,0 & & \\
\hline \multirow[t]{3}{*}{34} & Total & 0,0 & 11,5 & 21,2 & 30,8 & 36,5 & 5,13 & 0,1589 \\
\hline & Ingeniería & 0,0 & 15,6 & 21,9 & 37,5 & 25,0 & & \\
\hline & Traducción & 0,0 & 5,0 & 20,0 & 20,0 & 55,0 & & \\
\hline \multirow[t]{3}{*}{35} & Total & 0,0 & 11,5 & 15,4 & 50,0 & 23,1 & 4,03 & 0,2565 \\
\hline & Ingeniería & 0,0 & 6,3 & 12,5 & 59,4 & 21,9 & & \\
\hline & Traducción & 0,0 & 20,0 & 20,0 & 35,0 & 25,0 & & \\
\hline \multirow[t]{3}{*}{36} & Total & 1,9 & 9,6 & 17,3 & 36,5 & 34,6 & 3,61 & 0,4748 \\
\hline & Ingeniería & 3,1 & 6,3 & 12,5 & 37,5 & 40,6 & & \\
\hline & Traducción & 0,0 & 15,0 & 25,0 & 35,0 & 25,0 & & \\
\hline
\end{tabular}

36, el $71,1 \%$ de la muestra piensa que sus mayores errores en la escritura se encuentran en los aspectos formales de la lengua, esto es, gramática y puntuación.

Con la finalidad de conocer si existen diferencias en las percepciones de los estudiantes hacia la escritura académica, se compararon las respuestas de los dos grupos (Ciencias Humanas y Ciencias de la Ingeniería) en las cinco dimensiones del cuestionario. $\mathrm{Al}$ respecto, se obtuvo diferencias estadísticamente significativas en las dimensiones de "Revisión" y de “Textualización”.
En la Figura 1 se observa que la diferencia en la dimensión de "Revisión", entre ambos grupos, es estadísticamente significativa, con un valor $p=0,0028$. Dichos resultados evidencian que los estudiantes de Traducción son conscientes y otorgan mayor relevancia al proceso de revisión textual. Específicamente, se presentan diferencias significativas, a nivel estadístico, en relación con: la revisión del borrador de texto para hacerlo más organizado; con la dedicación de un tiempo para examinar el texto y con la revisión de las ideas expresadas, cuando estas no reflejan sus intenciones. En cambio, los de Ingeniería no acostumbran a dedicar 


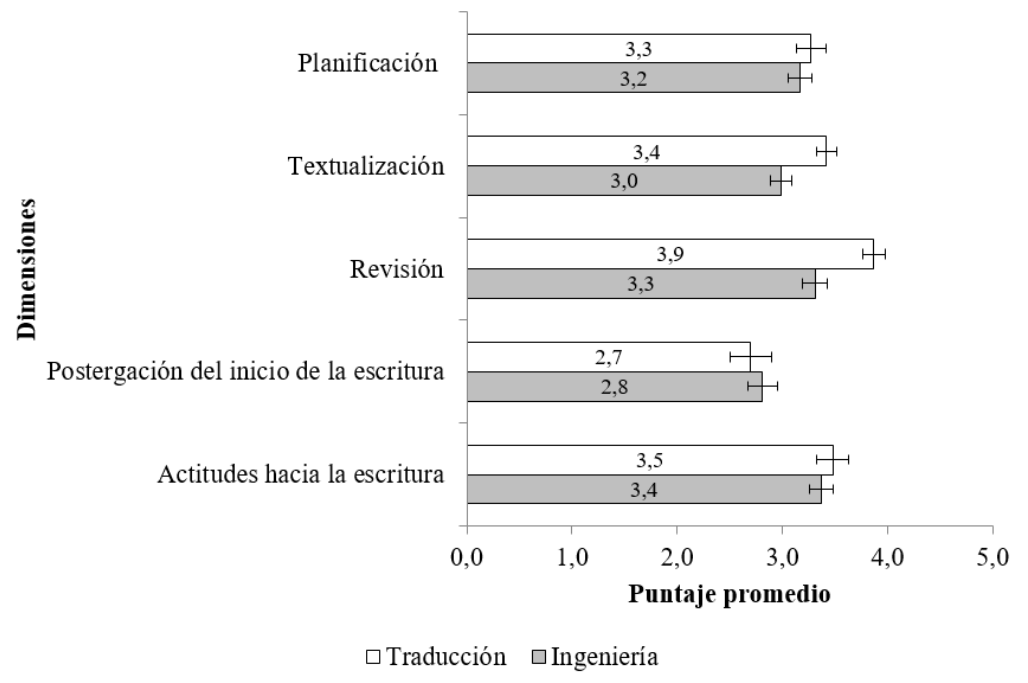

Figura 1 Diferencias entre los grupos en las distintas dimensiones del cuestionario. Las marcas que sobresalen cada una de las barras corresponden al error estándar

tiempo a la revisión y con frecuencia su borrador se constituye en el producto final.

Respecto a la dimensión de "Textualización”, se obtuvieron diferencias significativas entre ambos grupos, con un valor $p=0,0055$. Esto refleja que los alumnos de Ingeniería tienen mayores problemas para llevar a cabo el proceso de escribir o plasmar sus ideas en el papel. Los alumnos de Traducción reconocen que no tienen mayores dificultades para conectar los párrafos en un texto, mientras que un elevado porcentaje de estudiantes de Ingeniería lo asume. Del mismo modo, los alumnos de Ingeniería afirman que tienen nulos conocimientos acerca de las secuencias textuales que debe presentar un texto académico, dependiendo del género que corresponda; por su parte, el $75 \%$ de Traducción está de acuerdo con que las conoce. Pese a ello, convendría contrastar este estudio perceptivo con producciones textuales de los estudiantes; de esta manera, se podría corroborar si existe alguna correlación entre las concepciones de los alumnos hacia la escritura académica y lo que son capaces de realizar a la hora de escribir un texto.

\section{Conclusiones}

La alfabetización académica debería ser uno de los ejes centrales de las universidades para formar profesionales capaces de comunicarse e insertarse adecuadamente en los contextos disciplinares (académicos y profesionales) de su especialidad. Para implementar un programa de alfabetización académica se tiene que avanzar de manera gradual, primero, con cursos de formación general en el proceso de escritura y, luego, con asignaturas diferenciadas por área. No obstante, es preciso señalar que no se trata de restar protagonismo a las asignaturas de especialidad, sino de aunar voluntades, y optimizar el tiempo y los recursos disponibles para lograr los objetivos.

Los resultados obtenidos en este trabajo muestran distintas percepciones acerca de la escritura académica, según el área de formación de los estudiantes. En términos generales, es posible concluir que los alumnos de Ingeniería de la universidad donde se desarrolló el estudio perciben mayores dificultades que los de Traducción en el proceso de 
escritura, específicamente, en las dimensiones referidas a la textualización o escritura misma del texto, y a la revisión. Esto significa, por una parte, que los estudiantes de Ingeniería carecen de conocimientos sobre cómo elaborar un párrafo, tanto desde un punto de vista formal (por ejemplo, uso de conectores) como de contenido (tipologías textuales) y, por otra, no dedican tiempo a la revisión de su escrito. Si bien los alumnos de Traducción otorgan un espacio a la revisión, esta se limita a cuestiones formales, por lo que solo corrigen el texto en un nivel superficial.

Algunas de las limitaciones del presente estudio están en relación, por un lado, con la concepción general de escritura que se aborda en el cuestionario y que impide esbozar conclusiones acerca de prácticas escriturales específicas en cada área (ej. géneros textuales específicos) y, por otro, con diversificar la aplicación del instrumento a estudiantes de distintos ámbitos académicos.

Una proyección interesante de este estudio perceptivo sería triangular esta información con resultados obtenidos por estudiantes de Ciencias Humanas y Ciencias de la Ingeniería en la producción de textos académicos. Asimismo, sería pertinente indagar en las concepciones que tienen los docentes que imparten estos cursos sobre la escritura, ya que estas influyen directamente en las prácticas de los alumnos (Molano y López, 2007).

A partir de la información lograda en este estudio, coincidimos con Errázuriz et al. (2015) en que se necesita aumentar considerablemente el número de horas dedicadas a la escritura en las diferentes disciplinas, puesto que una o dos asignaturas a lo largo de la formación académica no son suficientes. También se pueden utilizar metodologías de enseñanza apoyadas por la tecnología (b-learning), donde se potencie la escritura de textos académicos de manera colaborativa (Figueroa y Aillon, 2015). Asimismo, se pueden proponer cursos de nivelación que apoyen a los estudiantes en su inserción en la educación superior (Errázuriz et al., 2015).
Si bien hay iniciativas de este tipo en la universidad donde se realizó el estudio, los esfuerzos se han concentrado en la habilidad de comprensión lectora y matemática, más que en la producción escrita, debido a las dificultades que implica el proceso de revisión de la escritura.

\section{Referencias}

Álvarez, G., y Difabio, H. (2017). Formación virtual en estrategias para la producción conceptual y escrita en el posgrado en Ciencias Sociales y Humanas. Revista Q, 10(20), 110-136. https://doi.org/10.18566/revistaq. v10n20.a05

Avilán, A. (2004). La escritura: abordaje cognitivo (Hacia una didáctica cognitiva de la escritura). Acción Pedagógica, 13(1), 31-48.

Bangert-Drowns, R., Hurley, M., y Wilkinson, B. (2004). The effects of school-based writing-to-learn interventions on academic achievement: A meta-analysis. Review ofEducationalResearch, 74(1),29-58.https:// doi.org/10.3102/00346543074001029

Bereiter, C., y Scardamalia, M. (1987). The psychology of written composition. Erlbaum.

Campbell, J., Smith, D., y Brooker, R. (1998). From conception to performance: How undergraduate students conceptualize and construct essays. Higher Education, 36(4), 449-469. https://doi.org/10.1023/A:1003451627898

Carlino, P. (2003). Alfabetización académica: un cambio necesario, algunas alternativas posibles. Educere, 6(20), 409-420.

Carlino, P. (2004). El proceso de escritura académica: cuatro dificultades de la enseñanza universitaria. Educere, $8(26), 321-327$.

Carlino, P. (2005). Escribir, leer y aprender en la universidad. Fondo de Cultura Económica.

Castelló, M. (2002). De la investigación sobre el proceso de composición a la enseñanza de la escritura. Revista Signos, 35(51-52), 149-162. https:// doi.org/10.4067/ S0718-09342002005100011

Difabio, H. (2012). Hacia un inventario de escritura académica en el posgrado. Revista de Orientación Educacional, 26(49), 37-53.

Difabio, H. (2013). Evaluación de las concepciones de escritura académica en doctorandos en educación. Actualidades Investigativas en Educación, 13(3), 1-21. https://doi.org/10.15517/aie.v13i3.12040 
Errázuriz, M., Arriagada, L., Contreras, M., y López, C. (2015). Diagnóstico de la escritura de un ensayo de alumnos novatos de Pedagogía en el campus Villarrica UC, Chile. Perfiles Educativos, 37(150), 76-90. https://doi. org/10.22201/iisue.24486167e.2015.150.53163

Figueras, N., y Puig, F. (2013). Pautas para la evaluación del español como lengua extranjera. Edinumen.

Figueroa, B., y Aillon, M. (2015). Escritura académica de un ensayo mediado por el aprendizaje colaborativo virtual. Estudios Pedagógicos, 41(1), 79-91. https://doi. org/10.4067/S0718-07052015000100005

Flower, L., y Hayes, J. (1981). A cognitive process theory of writing. College Composition and Communication, 32(4), 365-387. https://doi.org/10.2307/356600.

Hounsell, D. (1984). Learning and essay writing. En Ference Marton, Dai Hounsell y Noel Entwistle (Eds.), The Experience of Learning (pp.103-123). Scottish Academic Press.

Lavelle, E. (1993). Development and validation of an inventory to assess processes in college composition. British Journal of Educational Psychology, 63(3), 489. 499. https://doi.org/10.1111/j.2044-8279.1993. tb01073.x

Lavelle,E.,yBushrow,K.(2007).Writingapproachesofgraduate students. Educational Psychology, 27(6), 807-822. https://doi.org/10.1080/01443410701366001

Lavelle, E., y Guarino, A. (2003). A multidimensional approach to understanding college writing processes. Educational Psychology, 23(3), 295-305. https:// doi.org/10.1080/0144341032000060138

Lonka, K. (1996). The writing process questionnaire. Helsinki: Department of Psychology, University of Helsinki.

Martínez, A. (2011). La evaluación de las lenguas: garantias y limitaciones. Ediciones Mágina.

Molano, L., y López, G. (2007). Concepciones de profesores y estudiantes sobre la escritura académica en la Universidad Icesi. Lenguaje, 35(1), 119-146. https://doi.org/10.25100/lenguaje.v35i1.4848

Montolío, E. (2000). Manual práctico de escritura académica. Ariel.

Narvaja, E., Di Stefano, M., y Pereira, C. (2013). La lectura y la escritura en la universidad. Eudeba.

Ortiz, E. (2011). La escritura académica universitaria: estado del arte. Íkala, Revista de Lenguaje y Cultura, 16(28), $17-41$.

Rodríguez, B., y García, L. (2015). Escritura de textos académicos: dificultades experimentadas por escritores noveles y sugerencias de apoyo. Revista de Investigación Educativa, (20), 249-265. https://doi. org/10.25009/cpue.v0i20.1332

Scardamalia, M., y Bereiter, C. (1992). Dos modelos explicativos de los procesos de composición escrita. Infancia y Aprendizaje, 15(58), 43-64. https://doi. org/10.1080/02103702.1992.10822332

Tapia, M. (2014). Los comentarios escritos: género orientado a la consecución de otro género en el proceso de la escritura académica. Onomázein, (30), 254-268. https://doi.org/10.7764/onomazein.30.21

Torrance, M., Thomas, G., y Robinson, E. (1994). The writing strategies of graduate research students in the social sciences. Higher Education, 27(3), 379-392. https://doi.org/10.1007/BF03179901

Villalón, R. (2010). Las concepciones de los estudiantes sobre la escritura académica. (Tesis para optar al grado de doctor). Universidad Autónoma de Madrid. Recuperada de https://repositorio.uam.es/ handle/10486/4865

Villalón, R., y Mateos, M. (2009). Concepciones del alumnado de secundaria y universidad sobre la escritura académica. Infancia y Aprendizaje, 32(2), 219-232

Zimmerman, B., y Bandura, A. (1994). Impact of self-regulatory influences on writing course attainment. American Educational Research Journal, 31(4), 845862. https://doi.org/10.3102/00028312031004845

Cómo citar este artículo: Vine-Jara, Ana E. (2020). La escritura académica: percepciones de estudiantes de Ciencias Humanas y Ciencias de la Ingeniería de una universidad chilena. Íkala, Revista de Lenguaje y Cultura, 25(2), 475-491. http://www.doi.org/10.17533/udea.ikala.v25n02a02 